\title{
NON-INVASIVE TOOLS TO ASSESS THE RISK OF VARICES-NEEDING-TREATMENT IN CIRRHOSIS SECONDARY TO HCV
}

\author{
Laima Alam, Farrukh Saeed \\ Pak Emirates Military Hospital/National University of Medical Sciences (NUMS) Rawalpindi Pakistan
}

\begin{abstract}
Objective: To assess the non-invasive biomarkers of fibrosis for predicting varices-needing-treatment in patients with cirrhosis secondary to chronic-hepatitis-C.

Study Design: Cross sectional comparative study.

Place and Duration of Study: Department of Gastroenterology, Pak Emirates Military Hospital Rawalpindi, from Jan 2017 to Dec 2017.

Methodology: A total of 153 patients aged 18-79 with cirrhosis, whether compensated or decompensated, secondary to chronic-hepatitis-C were enrolled. Relevant serum tests were used to calculate non-invasive fibrosis indices and their diagnostic performance to predict the presence of varices and varices-needing-treatment was calculated.

Results: King's score showed the best performance in detecting varices due to high positive predictive value of $96.4 \%$ and positive likelihood ratio of 2.4. Overall, all the non-invasive fibrosis indices exhibited good performance with positive predictive value $>85 \%$ but none could rule out the presence of varices with adequate reliability due to low negative-predictive-value $(<65 \%)$. King's score exhibited relatively higher positive-predictivevalue $(70 \%)$ and negative predictive value $(51.1 \%)$ and the lowest negative-likelihood-ratio (0.6) for predicting varices needing treatment. Taken together, none of the non-invasive biomarkers of fibrosis could predict the presence of varices-needing-treatment with adequate accuracy due to low positive-predictive-value $(<85 \%)$ and low negative-predictive-value $(<65 \%)$.

Conclusion: The calculated non-invasive biomarkers of fibrosis and their optimum cutoff values showed modest accuracy for predicting varices and varices-needing-treatment. These indices may be used as first-line screening method for segregation of clinically significant portal hypertension and high risk esophageal varices-needingtreatment but may not be able to replace the gold standards like Fibroscan liver and Hepatic Venous Pressure Gradient measurements.
\end{abstract}

Keywords: Cirrhosis, HCV, Non-invasive biomarkers, Varices, Varices-needing-treatment.

This is an Open Access article distributed under the terms of the Creative Commons Attribution License (http://creativecommons.org/licenses/by/4.0), which permits unrestricted use, distribution, and reproduction in any medium, provided the original work is properly cited.

\section{INTRODUCTION}

Liver Cirrhosis and its complications represent one of the major health problems affronting medical community today with HCV induced cirrhosis accounting to about $130-170$ million people worldwide, making about $2-3 \%$ of the global population ${ }^{1}$. In Pakistan the frequency of hepatitis $\mathrm{C}$ infection varies from $0.4-33.7 \%^{2}$ whereas that of $\mathrm{HBV}$ is about $5 \%{ }^{3}$. Cirrhosis induced Portal Hypertension is an established cause of the complications including esophageal varices, ascites, hypersplenism and hepatic encephalopathy ${ }^{4}$. Esophageal varices accompany $60-80 \%$ of the cirrhotic

Correspondence: Dr Laima Alam, Dept of Gastroenterology, Pak Emirates Military Hospital Rawalpindi Pakistan

Received: 28 Mar 2018; revised received: 30 Jan 2019; accepted: 13 Feb 2019 patients, with variceal bleed ranked as one of the most devastating events ${ }^{5}$. The mortality rate secondary to variceal bleed in cirrhotic patients has been found to be $10-20 \%{ }^{6}$ and is therefore important to screen for the varices right after the diagnosis of cirrhosis and provide prophylaxis by either endoscopic band ligation, beta blockers or both?.

Guidelines regarding the schedule of endoscopic detection of esophageal varices have been formulated and have increased the medical care expenses exponentially ${ }^{8}$ especially for countries like Pakistan where the accessibility to invasive procedures like endoscopy is limited. The use of non-invasive methods to detect the presence of esophageal varices can segregate low risk 
patients from those with high risk esophageal varices requiring therapeutic endoscopy.

These non-invasive methods include CT scans, Transient Elastography and Biomarkers. The biomarkers are further divided in to Direct and In-direct. Procollagen I and III, Type IV Collagen, Hyaluronic acid, Laminin, Collagenases, Gelatinase A and B, Tissue Inhibitors of Matrix Metalloprotinases, Trans-forming Growth Factor Beta 1 and alpha are considered the direct biomarkers whereas ALT, AST/ALT ratio (AAR), AST/Platelet ration (APRI), Forns index, King score, Lok index, FIB-4 score, FI score, Bonacini score and Platelet/ Spleen ratio are some of the indirect biomarkers for assessing liver fibrosis 9.

The rationale of the study is to use these indirect biomarkers of fibrosis, for being in-expensive and non-invasive, in assessment of liver fibrosis and prediction of varices-needing-treatment before embarking on more costly serum markers, Transient Elastography and invasive methods like OGD and liver biopsy in countries like Pakistan where the availability and the cost effectiveness are the main hurdles in health care system.

\section{METHODOLOGY}

This prospective cross sectional study was carried out in the department of Gastroenterology, Pak Emirates Military Hospital Rawalpindi from January 2017 to December 2017.

A sample of 153 patients, aged 18-79, with cirrhosis, whether compensated or decompensated, secondary to chronic hepatitis $\mathrm{C}$ virus (HCV) was enrolled through simple convenience sampling with a standard error of 0.04 and a relative standard error of 8.08 for a confidence level of 95\% using National Statistics Services Calculator. Written informed consents were obtained from all participants. Cirrhosis is defined as the histological development of regenerative nodules surrounded by fibrous bands in response to chronic liver injury that leads to portal hypertension and end stage liver disease ${ }^{10}$. For this study however, ultrasound was used to check for liver fibrosis and splenomegaly (normal size $130 \mathrm{~mm}$ ) i.e; the manifestations of cirrhosis. Compensated cirrhosis is broadly classified into compensated advanced chronic liver disease (cACLD) and clinically significant portal hypertension (CSPH). For cACLD, a hepatic venous pressure gradient (HVPG) $>5 \mathrm{mmHg}$ and Transient Elastography (TE) $>10-15 \mathrm{kPa}$ whereas, for CSPH, HVPG >10 $\mathrm{mmHg}, \mathrm{TE} \geq 20-25 \mathrm{kPa}$ is required ${ }^{11}$. Decompensation occurs with the presence of acsites, encephalopathy, previous upper GI bleed and a raised bilirubin. Patients with pregnancy, lactation, immunosupression (HIV, malignancy), cirrhosis secondary to HBV, NASH, hemochromatosis, Primary Sclerosing Cholangitis and Primary Biliary Cirhosis, pacemaker insertion or defibrillator, liver transplant, drugs and infections causing deranged LFTs and thrombocytopenia and age $<18$ and $>79$ years were all excluded.

The enrolled patients had a baseline assessment including a history of cirrhosis, symptom duration, history of upper GI bleed, encephalopathy or ascites, drugs used, previous upper GI endoscopic study and a thorough medical and surgical history. A complete physical examination, baseline biochemical profile, blood indices, ultrasound scan for liver fibrosis and spleen size and an upper GI endoscopy for assessing the presence and grade of esophageal varices was offered to all patients. Using these variables APRI score, FIB-4, Lok index, King's score, Platelet/ Spleen ratio, cirrhosis discriminate score (CDS) and Goteborg University cirrhosis index (GUCI) are calculated using the standard formulae as below.

APRI: AST/upper limit of normal (considered as $40 \mathrm{IU} / \mathrm{L}) /$ platelet count $\left(10^{9} / \mathrm{L}\right)^{12}$

Lok Index: [exp (log odds)] / [1 $+\exp (\log$ odds) $]^{13}$

log odds: $-5.56-0.0089 \times$ number of platelets $\left(10^{3} / \mathrm{mm}^{3}\right)+1.26 \times($ AST $/$ ALT $)+5.27 \times \mathrm{INR}^{13}$.

King's score: Age (years) $\times$ AST $(\mathrm{U} / \mathrm{L}) \times \mathrm{INR} /$ number of platelets $(10 / \mathrm{L})^{14}$

Platelet/Spleen ratio: PLT (109/L) / Spleen diameter in $\mathrm{mm}^{15}$ 
FIB-4: Age (years) $\times$ AST $($ IU $/ L) /\left[\right.$ Plt $\left(10^{9} / \mathrm{L}\right) \times$ ALT(IU/L)1/2] ${ }^{15}$

CDS: Platelet score + ALT / AST + INR 16

GUCI: (AST/upper limit of normal AST) $\times$ INR $\times 100 /$ Platelets $^{16}$

The interpretation of the non-invasive liver fibrosis indices was done using standard publications ${ }^{16}$.
Baveno Consensus Conference ${ }^{11}$. Medium varices with endoscopic red sign (ERS) and large varices that required esophageal variceal band ligation (EVBL) were termed as varices-needing-treatment.

Data was statistically described in terms of mean \pm standard deviation (SD) for continuous data, frequencies (number of cases) and percentages when appropriate. Independent sample

Table-I: Baseline characteristics of patients with varices versus without varices and varices-not-needingtreatment vesus varices-needing-treatment.

\begin{tabular}{|c|c|c|c|c|c|c|}
\hline Variables & $\begin{array}{c}\text { No Varices } \\
(\mathrm{n}=11)\end{array}$ & $\begin{array}{l}\text { With Varices } \\
(n=120)\end{array}$ & $\begin{array}{c}p- \\
\text { value }\end{array}$ & $\begin{array}{l}\text { Varices not needing } \\
\text { treatment }(n=38)\end{array}$ & $\begin{array}{l}\text { Varices needing } \\
\text { treatment }(n=82)\end{array}$ & $\begin{array}{c}p- \\
\text { value }\end{array}$ \\
\hline $\operatorname{Sex}(M / F)(\%)$ & $\begin{array}{c}4(36.4) / \\
7(63.6)\end{array}$ & $\begin{array}{c}74(62) / \\
46(38)\end{array}$ & 0.10 & $\begin{array}{c}24(63.2) / \\
14(36.8)\end{array}$ & $\begin{array}{c}50(61) / \\
32(39)\end{array}$ & 0.82 \\
\hline Age (years) & $56 \pm 12$ & $55.5 \pm 9$ & 0.89 & $55.7 \pm 8.1$ & $55.4 \pm 9.5$ & 0.88 \\
\hline $\mathrm{Hb}(\mathrm{g} / \mathrm{dl})$ & $12.2 \pm 2.3$ & $10.5 \pm 2.5$ & 0.025 & $11.8 \pm 2$ & $9.86 \pm 2.4$ & $\leq 0.001$ \\
\hline TLC & $7.3 \pm 3$ & $5.3 \pm 2.2$ & 0.02 & $5.8 \pm 2.4$ & $5.1 \pm 2.1$ & 0.15 \\
\hline Plt & $188 \pm 117$ & $108.6 \pm 62$ & 0.002 & $129 \pm 83$ & $99 \pm 46.8$ & 0.02 \\
\hline $\operatorname{ALT}(\mathrm{U} / \mathrm{L})$ & $45.5 \pm 29.4$ & $65.8 \pm 52$ & 0.028 & $68.2 \pm 41$ & $64.7 \pm 56.6$ & 0.41 \\
\hline AST (U/L) & $48.7 \pm 45.3$ & $52.3 \pm 43$ & 0.13 & $53.1 \pm 35$ & $52 \pm 46.4$ & 0.76 \\
\hline Bilirubin (umol/L) & $17 \pm 14$ & $29 \pm 29.5$ & 0.03 & $24 \pm 18$ & $31.2 \pm 33.5$ & 0.75 \\
\hline $\begin{array}{l}\text { Alkaline } \\
\text { Phosphatase (U/L) }\end{array}$ & $253 \pm 117$ & $312 \pm 154$ & 0.25 & $336 \pm 160$ & $303 \pm 151.3$ & 0.16 \\
\hline Albumin (g/dl) & $37 \pm 0.4$ & $34 \pm 6.3$ & 0.11 & $36 \pm 4$ & $33 \pm 6.3$ & 0.04 \\
\hline INR & $1.3 \pm 0.4$ & $1.3 \pm 0.3$ & 0.79 & $1.2 \pm 0.2$ & $1.3 \pm 0.3$ & 0.30 \\
\hline Urea (umol/L) & $5.5 \pm 5.7$ & $8 \pm 12.7$ & 0.13 & $6.6 \pm 3.9$ & $8.7 \pm 15.1$ & 0.94 \\
\hline Creatinine (umol/L) & $73.7 \pm 19.2$ & $89.5 \pm 47.1$ & 0.36 & $89.4 \pm 35$ & $89.6 \pm 52$ & 0.64 \\
\hline Sodium $(\mathrm{mEq} / \mathrm{L})$ & $136 \pm 1.5$ & $137.7 \pm 2.5$ & 0.02 & $138 \pm 2.3$ & $137 \pm 2.6$ & 0.83 \\
\hline Potassium $(\mathrm{mEq} / \mathrm{L})$ & $4.2 \pm 1.5$ & $4.4 \pm 0.3$ & 0.14 & $4.4 \pm 0.3$ & $4.4 \pm 0.3$ & 0.6 \\
\hline MELD Na & $9.8 \pm 7.8$ & $10.9 \pm 2.7$ & 0.05 & $10.9 \pm 2.3$ & $10.82 \pm 2.9$ & 0.68 \\
\hline Spleen size $(\mathrm{mm})$ & $115 \pm 22.6$ & $142 \pm 23$ & 0.001 & $132 \pm 22$ & $147.2 \pm 22$ & 0.004 \\
\hline \multicolumn{7}{|c|}{ Child Pugh Turcott Score (\%) } \\
\hline A & $10(90.9)$ & $76(62.8)$ & \multirow{3}{*}{0.10} & $30(78.9)$ & $46(56.1)$ & \multirow{3}{*}{0.026} \\
\hline B & - & $36(30)$ & & $8(21.1)$ & $28(34.1)$ & \\
\hline C & $1(9.1)$ & $8(7)$ & & - & $8(9.8)$ & \\
\hline
\end{tabular}

Table-II: Baseline performance of non-invasive biomarkers of fibrosis in the enrolled patients.

\begin{tabular}{l|c|c|c|c|c|c} 
Variables & $\begin{array}{c}\text { No Varices } \\
(\mathbf{n = 1 1})\end{array}$ & $\begin{array}{c}\text { With Varices } \\
\mathbf{( n = 1 2 0 )}\end{array}$ & $\begin{array}{c}\boldsymbol{p} \text { - } \\
\text { value }\end{array}$ & $\begin{array}{c}\text { Varices not needing } \\
\text { treatment } \mathbf{( n = 3 8 )}\end{array}$ & $\begin{array}{c}\text { Varices needing } \\
\text { treatment }(\mathbf{n = 8 2})\end{array}$ & $\begin{array}{c}p \text { - } \\
\text { value }\end{array}$ \\
\hline APRI & $0.99 \pm 1.1$ & $1.76 \pm 2.3$ & 0.01 & $1.47 \pm 1.01$ & $1.89 \pm 2.7$ & 0.33 \\
\hline Lok Index & $0.6 \pm 0.2$ & $0.67 \pm 0.2$ & 0.31 & $0.61 \pm 0.2$ & $0.69 \pm 0.2$ & 0.04 \\
\hline King Score & $39.4 \pm 76.2$ & $44.95 \pm 59.2$ & 0.01 & $34.5 \pm 24.3$ & $49.8 \pm 69.3$ & 0.26 \\
\hline Platelet / Spleen & $1607 \pm 824$ & $807.52 \pm 546$ & $\leq 0.001$ & $1015 \pm 716$ & $711 \pm 417$ & 0.004 \\
\hline Fib 4 & $3.1 \pm 3.7$ & $4.02 \pm 2.9$ & 0.02 & $3.3 \pm 1.96$ & $4.3 \pm 3.2$ & 0.07 \\
\hline CDS & $6.27 \pm 1.6$ & $6.49 \pm 1.4$ & 0.69 & $6.13 \pm 1.4$ & $6.66 \pm 1.4$ & 0.06 \\
\hline GUCI & $1.72 \pm 2.9$ & $2.12 \pm 2$ & 0.014 & $1.72 \pm 1.2$ & $2.31 \pm 2.3$ & 0.25
\end{tabular}

APRI Aspartate aminotransferase to Platelet Ratio Index, CDS Cirrhosis Discriminate Score, GUCI Goteborg University Cirrhosis Index, $p \leq 0.05$ is considered significant

Small varices referred to size $<3 \mathrm{~mm}$, medium $3-5 \mathrm{~mm}$ and large $>5 \mathrm{~mm}$ as proposed at the t-test and Mann Whitney U-test were used to compare quantitative data whereas chi-square 
test was used to compare qualitative data such as gender and CTP score. The diagnostic performance of each non-invasive test of fibrosis in detecting varices and varices needing-treatment was assessed by using a receiver operating characteristic (ROC) analysis and each area under the curve (AUC) was calculated. Youden index was used to choose the optimal cutoff values. Sensitivity, specificity, positive predictive value (PPV), negative predictive value (NPV), positive likelihood ratio (+LR) and negative likelihood ratio (-LR) were used to calculate the diagnostic value of each non-invasive test.
The diagnostic performance of the non-invasive biomarkers of fibrosis with calculated cutoff values for predicting the presence of varices showed that King's score, APRI, Platelet/Spleen Ratio and FIB-4 exhibited the best performance as indicated by the AUCs of $0.73 \pm 0.09,0.73 \pm 0.1$, $0.73 \pm 0.09$ and $0.71 \pm 0.09$ respectively. King's score showed the best performance due to high PPV of $96.4 \%$ and +LR of 2.4. Overall, all the noninvasive fibrosis indices exhibited good performance with PPV $>85 \%$ but none could rule out the presence of varices with adequate reliability due to low NPV (<65\%) (figure, table-III).

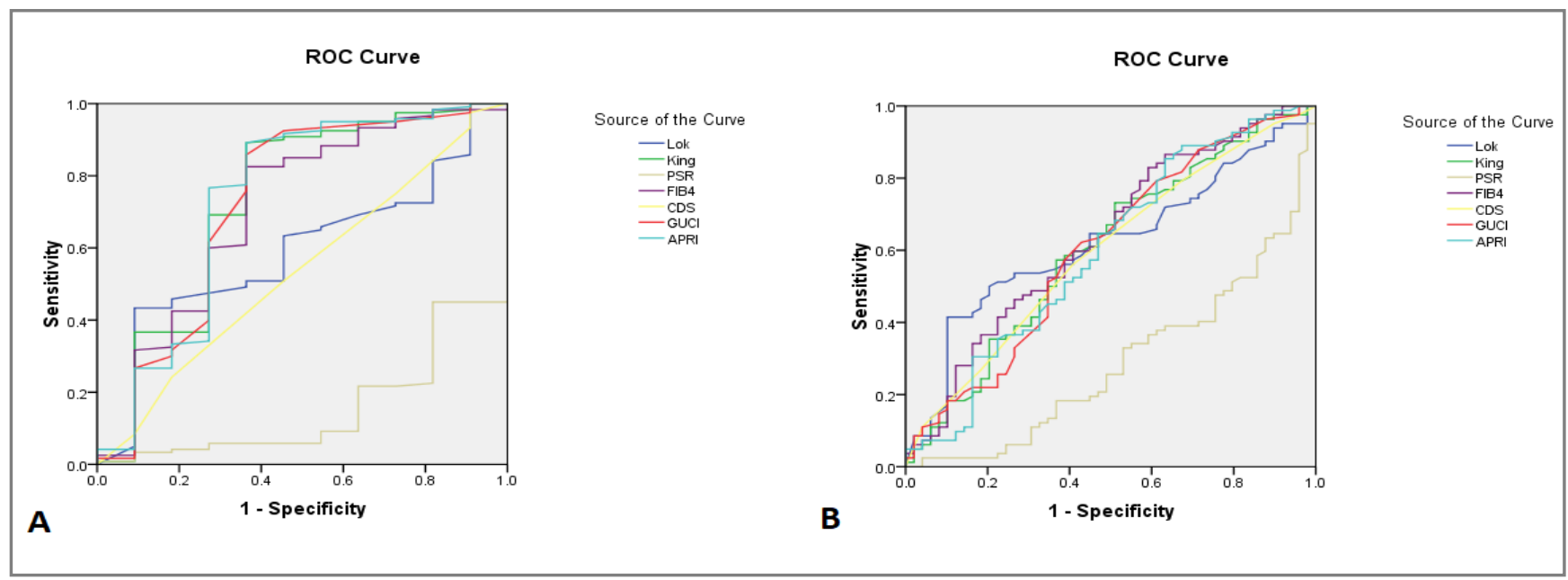

Figure: Performance of non-invasive biomarkers for the prediction of presence of A) varices and B) varicesneeding-treatment in cirrhosis. ROC curves showing the diagnostic accuracy of APRI, Lok index, King's score, FIB-4, Platelet/ Spleen Ratio, CDS and GUCI for optimum cutoff values.

All statistical analyses were performed using SPSS-19 software. All $p$-values $\leq 0.05$ were considered statistically significant.

\section{RESULTS}

A total of 153 patients were enrolled fulfilling the inclusion criteria, of whom 22 were found to be wrongly labeled as cirrhosis. The remaining 131 patients included 78 males and 53 females. Of these patients, 11 had no varices but varying grades of portal hypertensive gastropathy only, 38 had varices not needing treatment (small and medium) and 82 had varices needing treatment (medium and large). The baseline characteristics of the patients are summarized in the table-I \& II.
The optimized cutoff values and diagnostic performance for the non-invasive biomarkers of fibrosis for the prediction of varices-needingtreatment showed that King's score, Lok index, APRI and Fib-4 exhibited the best performance as indicated by AUCs; $0.6 \pm 0.05,0.6 \pm 0.5,0.6 \pm 0.05$ and $0.6 \pm 0.05$ respectively. GUCI showed a high NPV of $68 \%(>65 \%)$ but a low PPV $(45.3 \%)$ and a high -LR (0.9). King's score exhibited relatively higher PPV (70\%) and NPV (51.1\%) and the lowest -LR (0.6). Taken together, none of the noninvasive biomarkers of fibrosis could predict the presence of varices-needing-treatment with adequate accuracy due to low PPV $(<85 \%)$ and low NPV $(<65 \%)$ (table-IV, figure). 
Table-III: Performance of non-invasive biomarkers in prediction of presence of varices.

\begin{tabular}{l|c|c|c|c|c|c|c}
\hline & APRI & Lok index & $\begin{array}{c}\text { King's } \\
\text { Score }\end{array}$ & FIB-4 & $\begin{array}{c}\text { Platelets/ } \\
\text { Spleen Ratio }\end{array}$ & CDS & GUCI \\
\hline Cutoff & $\geq 0.81$ & $\geq 0.55$ & $\geq 13.4$ & $\geq 2$ & $<1065$ & $\geq 4.5$ & $\geq 0.9$ \\
\hline Sensitivity (\%) & 77.5 & 66 & 89.2 & 84 & 80 & 93 & 79 \\
\hline Specificity (\%) & 63.6 & 45.5 & 63.6 & 54.5 & 63.6 & 9.1 & 63.6 \\
\hline PPV (\%) & 96 & 93 & 96.4 & 95.3 & 96 & 92 & 96 \\
\hline NPV (\%) & 20.6 & 11 & 35 & 24 & 22.6 & 11 & 22 \\
\hline +LR & 2.08 & 1.2 & 2.4 & 1.8 & 2.1 & 1.0 & 2.1 \\
\hline -LR & 0.4 & 0.8 & 0.2 & 0.3 & 0.3 & 0.7 & 0.3 \\
\hline AUC (95\% CI) & $0.73 \pm 0.1$ & $0.6 \pm 0.8$ & $0.73 \pm 0.09$ & $0.71 \pm 0.09$ & $0.73 \pm 0.09$ & $0.53 \pm 0.09$ & $0.15 \pm 0.05$ \\
\hline
\end{tabular}

PPV Positive Predictive Value, NPV Negative Predictive Value, AUC Area Under curve, CI Confidence Interval, CDS Cirrhosis Discriminate Score, GUCI Goteborg University Cirrhosis Index, +LR Positive Likelihood Ratio, -LR Negative Likelihood Ratio.

Table-IV: Performance of non-invasive biomarkers in prediction of varices-needing-treatment (VNT).

\begin{tabular}{l|c|c|c|c|c|c|c} 
& APRI & Lok Index & $\begin{array}{c}\text { King's } \\
\text { Score }\end{array}$ & FIB-4 & $\begin{array}{c}\text { Platelets to } \\
\text { Spleen Ratio }\end{array}$ & CDS & GUCI \\
\hline Cutoff & $\geq 1.03$ & $\geq 0.62$ & $\geq 20$ & $\geq 3.07$ & $<843$ & $\geq 6.5$ & $\geq 1.02$ \\
\hline Sensitivity (\%) & 64.6 & 64.6 & 73.2 & 60 & 68.3 & 64.6 & 56 \\
\hline Specificity (\%) & 49 & 53 & 47 & 59 & 53 & 59 & 49 \\
\hline PPV (\%) & 67.9 & 70 & 70 & 71 & 71 & 70 & 45.3 \\
\hline NPV (\%) & 45.3 & 47.3 & 51.1 & 47 & 50 & 44.6 & 68 \\
\hline +LR & 1.3 & 1.4 & 1.4 & 1.5 & 1.4 & 1.6 & 1.1 \\
\hline -LR & 0.7 & 0.7 & 0.6 & 0.7 & 0.6 & 0.6 & 0.9 \\
\hline AUC (CI 95\%) & $0.6 \pm 0.05$ & $0.6 \pm 0.5$ & $0.6 \pm 0.05$ & $0.6 \pm 0.05$ & $0.3 \pm 0.04$ & $0.6 \pm 0.05$ & $0.6 \pm 0.05$ \\
\hline
\end{tabular}

PPV Positive Predictive Value, NPV Negative Predictive Value, AUC Area Under curve, CI Confidence Interval, CDS Cirrhosis Discriminate Score, GUCI Goteborg University Cirrhosis Index, +LR Positive Likelihood Ratio, -LR Negative Likelihood Ratio.

\section{DISCUSSION}

Identification of high risk esophageal varices, offering prompt therapy and keeping a follow up plan of upper GI endoscopy is of utmost importance in managing cirrhotic patients. Since the procedure is not cost effective and is invasive, the need for careful selection of patients with relatively inexpensive and non-invasive biomarkers to restrict unnecessary endoscopies is required. This study uses non-invasive biomarkers of liver fibrosis to predict the presence of varices and those varices that require EVBL as treatment using APRI, Lok index, King's score, FIB-4, Platelet/Spleen ratio, CDS and GUCI.

The diagnostic performance of APRI for predicting varices with a cutoff of $\geq 0.81$ is higher for this study as compared to a similar study ${ }^{17}$ but same as other studies ${ }^{16}$. For a cutoff of $\geq 1.3$, the study has a low diagnostic performance for predicting high risk varices needing treatment as compared to similar studies ${ }^{17,18}$ but same as another study ${ }^{19}$. Lok index with cutoff values of $\geq 0.55$ and $\geq 0.62$ for predicting presence of varices and varices needing treatment shows lower diagnostic performance as compared to other comparative studies ${ }^{16-18}$, but has shown good diagnostic performance for detection of varices in this study. King's score with a cutoff of $\geq 13.4$ and $\geq 20$ for predicting varices and varices needing treatment shows a relatively good diagnostic performance with high sensitivity, specificity, PPV, NPV, +LR and a low-LR in comparison to a similar study ${ }^{17}$. FIB-4 with a cutoff of $\geq 3.07$ for detection of varices needing treatment showed superior diagnostic performance as compared to a similar study 20 but in contrast to another study ${ }^{21}$. Platelet/spleen ratio predicted the presence of varices adequately but the cutoff used for detecting varices needing treatment showed lower diagnostic performance in comparison to a similar study ${ }^{21}$. CDS showed a modest diagnostic performance for both the groups of patients in contrast to GUCI that poorly predicted the presence of varices. The results were in accordance to a similar study ${ }^{22}$.

In summary, these non-invasive markers of fibrosis could predict the presence of varices with 
modest accuracy due to high PPV but the NPV was low for all the tests used, whereas the diagnostic performance for predicting varices-needing-treatment was low, which was in accordance to similar studies ${ }^{16-21}$.

\section{CONCLUSION}

The calculated non-invasive biomarkers of fibrosis and their optimum cutoff values showed modest accuracy for predicting varices and varices needing treatment. These indices may be used as first line screening method for segregation of clinically significant portal hypertension and high risk esophageal varices needing treatment but may not be able to replace the gold standards like Fibroscan liver and Hepatic Venous Pressure Gradient measurements.

\section{CONFLICT OF INTEREST}

This study has no conflict of interest to be declared by any author.

\section{REFERENCES}

1. Cuadros DF, Branscum AJ, Miller FD, Abu-Raddad LJ. Spatial epidemiology of hepatitis $C$ virus infection in Egypt: analyses and implications. Hepatology 2014; 60(4): 1150-59.

2. Umer $M$, Iqbal $M$. Hepatitis $C$ virus prevalence and genotype distribution in Pakistan: Comprehensive review of recent data. World J Gastroenterol 2016; 22(4): 1684-700.

3. Ali M, Idrees M, Ali L, Hussain A, Rehman IU, Saleem S, et al. Hepatitis B virus in Pakistan: A systematic review of prevalence, risk factors, awareness status and genotypes. Virol J 2011; 8(1): 102-10.

4. Zhang W, Wang L, Wang L, Li G, Huang A, Yin P, et al. Liver stiffness measurement, better than APRI, Fibroindex, Fib-4, and NBI gastroscopy, predicts portal hypertension in patients with cirrhosis. Cell Biochem Biophys 2015; 71(2): 865-73.

5. Ferreira CN, Seijo S, Plessier A, Silva-Junior G, Turon F, Rautou $P$, et al. Natural history and management of esophagogastric varices in chronic noncirrhotic, nontumoral portal vein thrombosis. Hepatology 2016; 63(5): 1640-50.

6. deFranchis R. Expanding consensus in portal hypertension: Report of the Baveno VI Consensus Workshop: Stratifying risk and individualizing care for portal hypertension. J Hepatol 2015; 63(3): 743-52.

7. Gupta A, Mehta A, Mishra N, Bansal R, Yadav A. Effectiveness of transjugular intrahepatic portosystemic shunt in variceal bleeding: an audit from a tertiary care center in north India. J ClinInterv Radiol ISVIR 2017; 1(3): 150-55.

8. Hassan EA, El-Rehim ASE, Sayed ZEA, Ashmawy AM, Kholef EFM, Sabry A, Elsewify WE. Noninvasive fibrosis scores as prognostic markers for varices needing treatment in advanced compensated liver cirrhosis. Open J Gastroenterol 2017; 7(8): 1-5.

9. Fallatah H. Noninvasive biomarkers of liver fibrosis: an overview. Adv Hepatol 2014; 2014: 1-15.

10. EASL-ALEH Clinical Practice Guidelines: Non-invasive tests for evaluation of liver disease severity and prognosis. J Hepatol 2015; 63(1): 237-64.

11. de Franchis R. Expanding consensus in portal hypertension. Report of the Baveno VI Consensus Workshop: Stratifying risk and individualizing care for portal hypertension. J Hepatol 2015; 63(3): 743-52.

12. Cui J, Ang B, Haufe W, Hernandez C, Verna E, Sirlin C, et al. Comparative diagnostic accuracy of magnetic resonance elastography vs. eight clinical prediction rules for non-invasive diagnosis of advanced fibrosis in biopsy-proven non-alcoholic fatty liver disease: a prospective study. Aliment Pharmacol Ther 2015; 41(12): 1271-80.

13. Attallah A, Omran D, Omran M, Albannan M, Zayed R, Saif S, et al. Fibro-Mark: a panel of laboratory parameters for predicting significant fibrosis in chronic hepatitis C patients. Br J Biomed Sci 2017; 75(1): 19-23.

14. Díaz-Piedra P, Olay-Fuentes G, Hernández-Gómez R, Cervantes-Villagrana RD, Presno-Bernal JM, Alcántara-Gómez LE. Determinación de los intervalos de referencia de biometría hemática poblaciónmexicana. Rev Latinoamer Patol Clin 2012; 59(4): 243-50.

15. Xiao G, Yang J, Yan L. Comparison of diagnostic accuracy of aspartate aminotransferase to platelet ratio index and fibrosis- 4 index for detecting liver fibrosis in adult patients with chronic hepatitis B virus infection: A systemic review and meta-analysis. Hepatology 2014; 61(1): 292-302.

16. Wang L, Feng Y, Ma X, Wang G, Wu H, Xie X, et al. Diagnostic efficacy of noninvasive liver fibrosis indexes in predicting portal hypertension in patients with cirrhosis. PLoS One 2017; 12(8): e0182969.

17. Deng H, Qi X, Peng Y, Li J, Li H, Zhang Y, et al. Diagnostic accuracy of APRI, AAR, FIB-4, FI, and king scores for diagnosis of esophageal varices in liver cirrhosis: a retrospective study. Med Sci Monit 2015; 21(1): 3961-77.

18. Jangouk P, Turco L, De Oliveira A, Schepis F, Villa E, GarciaTsao G. Validating, deconstructing and refining Baveno criteria for ruling out high-risk varices in patients with compensated cirrhosis. Liver Int 2017; 37(8): 1177-83.

19. de Mattos AZ, de Mattos AA, Daros LF, Musskopf MI. Aspartate aminotransferase-to-platelet ratio index (APRI) for the noninvasive prediction of esophageal varices. Ann Hepatol 2013; 12(5): 810-14.

20. Elalfy H, Elsherbiny W, Rahman AA, Elhammady D, Shaltout S, Elsamanoudy A, et al. Diagnostic non-invasive model of large risky esophageal varices in cirrhotic hepatitis $\mathrm{C}$ virus patients. World J Hepatol 2016; 8(24): 1028.

21. Stasi C, Milani S. Evolving strategies for liver fibrosis staging: The non-invasive assessment. World J Gastroenterol 2017; 23(2): 191-95.

22. Farid K, Omran M, Farag R, Arafa M, Emran T. Development and evaluation of a novel score for prediction of large oesophageal varices in patients with hepatitis c virus-induced liver cirrhosis. Br J Biomed Sci 2017; 74(3): 138-43. 